\title{
Adequate Neutrophil Responses and Non- Inferior Clinical Outcomes Can Be Achieved by a Two-Day Course of Low-Dose Filgrastim: A Retrospective Single Institution Experience
}

\author{
Raj Singh ${ }^{1}$, Henry Heisey ${ }^{2}$, Ahmed G. Elsayed ${ }^{3}$, Maria T. Tirona ${ }^{4}$
}

1. Department of Radiation Oncology, Virginia Commonwealth University, Richmond, USA 2. Student, Marshall University, Joan C. Edwards School of Medicine 3. Hematology and Oncology, Joan C. Edwards School of Medicine at Marshall University, Huntington, USA 4. Director of Medical Oncology, Marshall University, Joan C. Edwards School of Medicine

$\square$ Corresponding author: Ahmed G. Elsayed, gamal_masry@yahoo.com Disclosures can be found in Additional Information at the end of the article

\section{Abstract}

\section{Background}

Filgrastim is used in the setting of chemotherapy-induced neutropenia to stimulate recovery of bone marrow, which allows for further chemotherapy administration without delay. The recommended dose is $5 \mathrm{ug} / \mathrm{kg}$. The commercially available vials of the drug come in two strengths; $300 \mathrm{ug}$ and $480 \mathrm{ug}$. Due to these limitations in dosage formulations, it is a frequent occurrence to administer a lower dosage to patients weighing more than $60 \mathrm{~kg}$, in whom the ideal dose would have been more than $300 \mathrm{ug}$ but less than $480 \mathrm{ug}$. It is also a frequent practice to administer the drug for two consecutive days as it often leads to adequate response that will render patients eligible for their next cycle administration.

\section{Objective}

To determine whether a course of 300 ug of filgrastim administered daily for two consecutive days was as successful at reducing chemotherapy-induced neutropenia-related complications in patients with a higher weight (>60 kg) and hence receiving suboptimal dose as compared to those with weight less than $60 \mathrm{~kg}$ who are receiving the recommended dose.

\section{Methods}

Received 08/28/2017

Review began 11/07/2017 Review ended 12/06/2017 Published 12/19/2017

(C) Copyright 2017

Singh et al. This is an open access article distributed under the terms of the Creative Commons Attribution License CC-BY 3.0., which permits unrestricted use, distribution, and reproduction in any medium, provided the original author and source are credited.
We identified 91 patients from our facility with chemotherapy-induced neutropenia treated with 300 ug of filgrastim daily for two consecutive days, and we separated them into low, medium, and high weight groups. Multivariate logistic regression models examined correlations between outcomes (e.g., increases in absolute neutrophil count) and predictors (e.g., weight groups).

\section{Results}

The vast majority of encounters demonstrated rises in white blood cell (WBC) and absolute neutrophil count (ANC). Infection rates were not significantly different between low and medium weight groups ( $5 \%$ vs $0 \% ; \mathrm{p}=0.1658$ ), but the high weight group's infection rate was significantly higher than the medium weight group ( $5 \%$ vs $33 \% ; \mathrm{p}=0.001)$. The high weight

\section{How to cite this article}

Singh R, Heisey H, Elsayed A G, et al. (December 19, 2017) Adequate Neutrophil Responses and NonInferior Clinical Outcomes Can Be Achieved by a Two-Day Course of Low-Dose Filgrastim: A Retrospective Single Institution Experience. Cureus 9(12): e1968. DOI 10.7759/cureus.1968 
group did have an increased rate of febrile neutropenia as compared to medium and low weight groups, but these differences were not significant. Incidences of chemotherapy delay and dose reduction were comparable across the three weight groups.

\section{Limitations}

Retrospective study, small sample size, heterogeneous cancer sites and different chemotherapy regimens administered limit generalizability of findings.

\section{Conclusion}

Patients with weights $<85 \mathrm{~kg}$ receiving a two-day course of $300 \mathrm{ug}$ of filgrastim have similar neutropenia-related complication rates with a potential percent cost-savings of roughly $43 \%$.

Categories: Oncology, Quality Improvement

Keywords: chemotherapy, neutropenia, g-csf, neutropenic fever, cost savings, quality improvement

\section{Introduction}

Patients receiving chemotherapy for various malignancies often develop neutropenia and consequent infection resulting in increased morbidity and mortality, compromised treatment outcomes, and excess cost. More than 60,000 cancer patients undergoing chemotherapy are hospitalized for neutropenia each year in the US, with an average cost of $\$ 13,372$ per hospitalization with associated inpatient mortality [1, 2]. Filgrastim, a pharmaceutical analog of granulocyte colony-stimulating factor (G-CSF), is commonly used to prevent infection in immunocompromised cancer patients by stimulating production of neutrophils by the bone marrow [3].

The therapeutic role of G-CSF analogs in treating febrile neutropenia (FN) is debated. The Infectious Disease Society of America (IDSA) does not recommend G-CSF in the setting of neutropenic fever [4]. However, the role of G-CSF in preventing chemotherapy-induced neutropenia is more established. The American Society of Clinical Oncology (ASCO), the European Society for Medical Oncology (ESMO), the European Organization for Research and Treatment of Cancer (EORTC), and the National Comprehensive Cancer Network (NCCN) suggest consideration for therapeutic use in select patients, particularly those with a $20 \%$ or greater risk of developing FN [5-8].

For at least two decades, therapeutic application of G-CSF has been known to reduce the duration of neutropenia even in afebrile patients with severe neutropenia [9]. Colonystimulating factors like filgrastim have decreased the amount of time patients spend hospitalized, improved their ability to recover from neutropenia, and decreased the duration of antibiotic use, but research has not yet demonstrated a mortality benefit [10]. More recently, secondary intervention with G-CSF for neutropenia has been shown to increase subsequent achievement of adequate chemotherapy dose intensity [11].

Pragmatically, however, the high cost of G-CSF drugs underscores the importance of identifying opportunities for cost savings within the context of the common usage of the drugs. The effective annual per-patient drug cost of filgrastim has been estimated by budget impact models to be between $\$ 15,573$ and $\$ 30,663$ [12]. At our own institution, the average wholesale single dose of $300 \mathrm{ug} / \mathrm{mL}$ of filgrastim is $\$ 1080$ as compared to $\$ 1889$ for a $480 \mathrm{ug}$ vial. As such, patients with weights $>60 \mathrm{~kg}$ are often times billed for the $480 \mathrm{ug}$ dose despite the fact that they do not require the entire additional dose for the cost incurred. Generally, in our own institution, the same practice is followed, though sometimes patients with weights $>60 \mathrm{~kg}$ may receive 300 
ug of filgrastim rather than the recommended dose that would require a purchase of $480 \mathrm{ug}$ vial. Our facility frequently uses a two-day dosing regimen of G-CSF during subsequent chemotherapy cycles for those patients who had previous delays in their treatment due to neutropenia. As such, we aimed at determining if a two-day course of lower doses of filgrastim demonstrates efficacy for treating established neutropenia and achieves similar clinical outcomes that have previously been reported at the recommended dosage.

\section{Materials And Methods}

A retrospective chart review was performed to identify all patients with chemotherapy-induced neutropenia at our institution treated with two consecutive doses of 300 ug of filgrastim between September 13, 2011 and September 13, 2016. Patients were excluded if they did not have a recorded WBC and ANC within two days of the first dose of filgrastim administration. Based on these criteria, a total of 91 patients over 150 encounters were included in the analysis. Relevant patient information was collected and recorded for use as independent variables. These included age ( $<$ or $>70$ years), weight, race (Caucasian or non-Caucasian), estimated glomerular filtration rate (GFR, < or $>60$ ), primary malignancy site, and the chemotherapeutic agent(s) received.

Regarding weight, patients were divided into three primary groups: low (weight $<60 \mathrm{~kg}$ ), medium ( $60 \mathrm{~kg}<$ weight $<85 \mathrm{~kg}$ ), and high (weight greater than or equal to $85 \mathrm{~kg}$ ). This was done as patients with weights $<60 \mathrm{~kg}$ receiving $300 \mathrm{ug}$ of filgrastim were receiving the recommended $5 \mathrm{ug} / \mathrm{kg}$ dose. Given that the low weight group received the recommended dose, this cohort was treated as the control group for the purposes of this study. As patients in the medium and high weight groups were receiving a sub-therapeutic dose of filgrastim based on label recommendations (as patients with weights $>60 \mathrm{~kg}$ are recommended to be administered greater than 300 ug of filgrastim), these two cohorts served as the groups of interest. Clinical outcomes relating to chemotherapy-induced neutropenia were examined among the low and medium/high weight groups.

The primary outcomes of our analysis were examining complications relating to neutropenia, including FN, infections, hospitalizations, dose reductions, or missed subsequent chemotherapy cycles during the two weeks following treatment with filgrastim. Hospitalization relating to neutropenia was defined as hospitalization within two weeks of filgrastim treatment secondary to neutropenia, fever, or infection. Primary outcomes were obtained from laboratory findings dated from 1 to 22 days following treatment (mean 6.6 days, standard deviation 3.2 days). Other outcomes were obtained by review of oncologist documentation at our center during the two weeks following filgrastim administration.

Data analysis was performed using Stata 14.0 (StataCorp, College Station, TX). Multivariate logistic regressions were run to determine the effect that potential prognostic factors included in our study had on the probability of relevant outcomes previously discussed.

\section{Results}

A summary of the patient cohort studied can be found in Table 1 . The average weight of the cohort was $66.74 \mathrm{~kg}$ (range: $41.9-109.6 \mathrm{~kg}$ ). Thirty of 91 patients had weights less than or equal to $60 \mathrm{~kg}$ (low weight group) and thus received the recommended dose of filgrastim. The other 61 patients studied had weights greater than $60 \mathrm{~kg}$ and received less than the recommended dose, with 53 patients weighing between 60 and $85 \mathrm{~kg}$ (medium weight group) and eight patients with weights greater than $85 \mathrm{~kg}$ (high weight group). Twenty-four patients (26\%) were receiving chemotherapy for breast cancer, 16 for colorectal cancer (18\%), 33 for lung cancer (36\%), and the remaining 18 (20\%) for cancers of other sites. The mean total dose in ug/kg that was administered over the course of two days was 7.37 (SD: 2.28) for patients with weights >85 


\section{Cureus}

$\mathrm{kg}, 8.38$ (SD: 3.11) for those in the medium weight group, and 11.19 (SD: 4.58) for patients with weights $<60 \mathrm{~kg}$.

\section{Variable}

Total Patients

Patients with weight $\geq 85 \mathrm{~kg}$

Patients with $60 \mathrm{~kg}<$ weight $<85 \mathrm{~kg}$

Patients with weight $\leq 60 \mathrm{~kg}$

Total Filgrastim Administrations

Encounters with weight $\geq 85 \mathrm{~kg}$

Encounters with $60 \mathrm{~kg}<$ weight $<85 \mathrm{~kg}$

Encounters with weight $\leq 60 \mathrm{~kg}$

Average Dose by Weight Group (ug/kg)

Patients with weight $\geq 85 \mathrm{~kg}$

Patients with $60 \mathrm{~kg}<$ weight $<85 \mathrm{~kg}$

Patients with weight $\leq 60 \mathrm{~kg}$

Gender

Male

Female

Age (range)

Race

Caucasian

Hispanic

Asian-American

African-American

Unknown

Height (cm) (range)

Weight $(\mathrm{kg})$ (range)

$B M I$ (range)

GFR (range)

Malignancy Site

Breast

\section{Summary Statistics}

91 patients

8 patients

53 patients

30 patients

150 encounters

12 encounters

91 encounters

47 encounters

7.37 (SD: 2.28)

8.38 (SD: 3.11)

11.19 (SD: 4.58)

23

68

$60.64(33-87)$

73 patients

1 patient

1 patient

3 patients

13 patients

$165.79(149-185.4)$

$66.74(41.9-109.6)$

$24.31(16.03-41.8)$

74.49 (26.59 - 135.69)

24 patients 


\section{Cureus}

Colorectal

Lung

Other

Chemotherapy Received

Paclitaxel

Gemcitabine

Cisplatin

Carboplatin

Folfox

Doxorubicin

Bevacizumab

Etoposide
16 patients

33 patients

18 patients

24 patients

11 patients

16 patients

22 patients

21 patients

6 patients

14 patients

12 patients

\section{TABLE 1: Patient characteristics.}

BMI: Body mass index; GFR: Glomerular filtration rate.

Outcomes for the entire patient cohort studied can be found in Table 2. The average elapsed time between filgrastim administration and repeat complete blood count (CBC) was 6.63 days (range: 1 day to 22 days). Following administration of filgrastim, 98\% and $95.33 \%$ of encounters had documented rises in WBC and ANC, respectively. Patients in the low, medium, and high weight groups had similar WBC responses to filgrastim, as $95.74 \%, 98.9 \%$, and $100 \%$ of such encounters had documented rises in WBC, respectively. Similar results were noted for the low (95.74\%), medium (94.51\%), and high (100\%) weight groups with regards to ANC response rates. 


\section{Cureus}

\section{Variable}

Average number of days between filgrastim

administration and repeat $\mathrm{CBC}$ (range)

Encounters with rise in WBC

Encounters with rise in ANC

\section{Complication Rates}

Instances of infection within two weeks of encounter

Instances of FN-related hospitalization within two weeks

of encounter

Instances of patients having to miss next cycle of chemotherapy

Instances of patients having dose reduction

\section{Summary Statistics}

6.63 (1 to 22$)$

Weight $<60 \mathrm{~kg}-95.74 \% \quad 60 \mathrm{~kg}<$ Weight $<85 \mathrm{~kg}-98.9 \%$ Weight $>85 \mathrm{~kg}-100 \%$

Weight $<60 \mathrm{~kg}-95.74 \% 60 \mathrm{~kg}<$ Weight $<85 \mathrm{~kg}-94.51 \%$ Weight $>85 \mathrm{~kg}-100 \%$

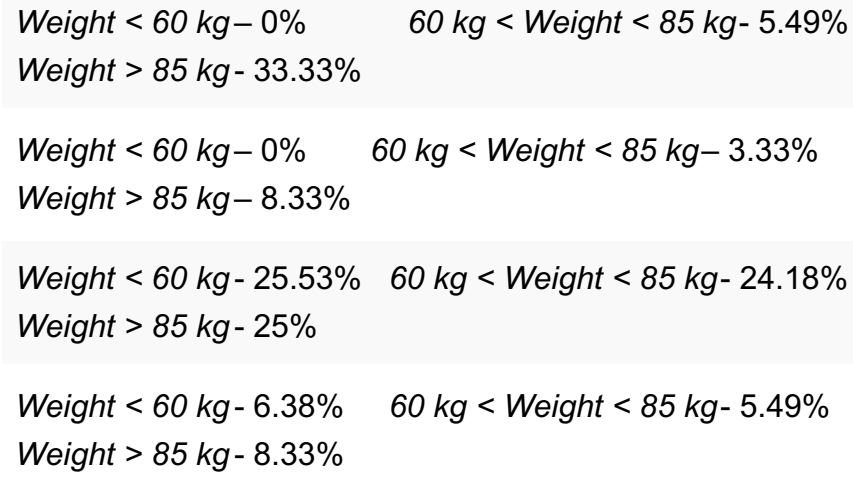

\section{TABLE 2: Summary of both laboratory and clinical responses over 150 patient encounters following administration of a shortened course of low-dose filgrastim.}

ANC: Absolute neutrophil count; CBC: Complete blood count; FN: Febrile neutropenia; WBC: White blood cell.

A summary of incidences of FN-related hospitalizations, incidences of infections, dose reductions, and treatment delays can also be found in Table 2. Patients in the high weight group were noted to have a markedly higher rate of infection two weeks following filgrastim administration (33\%) as compared to the low weight (0\%) and medium weight (5.49\%) groups. The association between having a high weight and an increased likelihood of infection was statistically significant ( $\mathrm{p}=0.001$; Table 3 ). A similar trend was found with regards to incidences of FN-related hospitalizations, which were highest among the higher weight group (8.33\%) as compared to the medium (3.33\%) and low weight groups (0\%). However, this was not found to be significant $(\mathrm{p}=0.239$ ). None of the prognostic factors included in our analysis were noted to be correlated with requiring hospitalization secondary to FN. Roughly $6 \%, 5.5 \%$, and $8 \%$ of patients in the low, medium, and high weight groups, respectively, required dose reductions due to neutropenia after receiving filgrastim. Weight was not correlated with incidences of dose reductions. Nearly $25 \%$ of patients in each weight group also experienced delays in treatment and none of the variables analyzed were significantly associated with treatment delays. 


\section{Cureus}

\begin{tabular}{|c|c|c|c|c|}
\hline Variable & $\begin{array}{l}\text { p-value (Febrile neutropenia-related } \\
\text { hospitalization) }\end{array}$ & $\begin{array}{l}\text { p-value } \\
\text { (Infection) }\end{array}$ & $\begin{array}{l}p \text {-value (Treatment } \\
\text { Delay) }\end{array}$ & $\begin{array}{l}\text { p-value (Dose } \\
\text { Reduction) }\end{array}$ \\
\hline $\begin{array}{l}60 \mathrm{~kg}<\text { Weight } \\
<85 \mathrm{~kg}\end{array}$ & 0.559 & 0.1658 & 0.863 & 0.147 \\
\hline Weight $\geq 85 \mathrm{~kg}$ & 0.239 & $0.001^{* *}$ & 0.978 & 0.724 \\
\hline Gender & 0.185 & 0.372 & 0.609 & 0.372 \\
\hline Age $>70$ & 0.962 & 0.898 & 0.620 & 0.503 \\
\hline $\begin{array}{l}\text { Race (Non- } \\
\text { Caucasian) }\end{array}$ & $0.98 \%$ & 0.169 & 0.412 & 0.893 \\
\hline$G F R<60$ & 0.962 & $0.034^{*}$ & 0.697 & 0.152 \\
\hline
\end{tabular}

TABLE 3: Multivariate logistic regressions examining impact of potential prognostic factors on neutropenia-related complications following filgrastim administration.

${ }^{*}=p<0.05,{ }^{* *}=p<0.01$.

FN: Febrile neutropenia; GFR: Glomerular filtration rate.

WBC and ANC responses for the cohort broken down by weight can be found in Table 4. A similar number of patients in the low, medium, and high weight groups had documented rises in WBC counts $(95.74 \%, 98.9 \%$, and 100\%) and ANC counts $(95.74 \%, 94.51 \%$, and 100\%, respectively) with all being significant increases $(\mathrm{p}<0.0001)$ following paired t-tests. 


\section{Cureus}

\begin{tabular}{|c|c|c|c|}
\hline Variable & Weight $\leq 60 \mathrm{~kg}$ & $60 \mathrm{~kg}<$ Weight $<85 \mathrm{~kg}$ & Weight $\geq 85$ kg \\
\hline \multicolumn{4}{|l|}{ WBC Counts } \\
\hline Average Pre-Filgrastim WBC (range) & 2270 (500 to 4500$)$ & 1832 (500 to 5200$)$ & $2258(1000$ to 4400$)$ \\
\hline Average Post-Filgrastim WBC (range) & 7302 (600 to 23,600$)$ & 7281 (900 to 36,300$)$ & $6508(2700$ to 12,500$)$ \\
\hline Average Rise in WBC (range) & $5032(-600$ to 21,000$)$ & $5389(-1300$ to 34,200$)$ & 4250 (100 to 10,600$)$ \\
\hline $\begin{array}{l}\text { Average Percent Change in WBC } \\
\text { (range) }\end{array}$ & $352 \%(77 \%$ to $14,000 \%)$ & $494 \%(41 \%$ to $18,686 \%)$ & $348 \%(103 \%$ to $6800 \%)$ \\
\hline Encounters with rise in WBC & $95.74 \%$ & $98.9 \%$ & $100 \%$ \\
\hline \multicolumn{4}{|l|}{ ANC Counts } \\
\hline Average Pre-Filgrastim ANC (range) & $675(100$ to 1500$)$ & $638(84$ to 1600$)$ & $950(200$ to 1900$)$ \\
\hline Average Post-Filgrastim ANC (range) & 4801 (300 to 20,500$)$ & 5173 (307 to 33,000$)$ & $4943(1400$ to 10,100$)$ \\
\hline Average Rise in ANC (range) & $4127(-600$ to 19,900$)$ & $4534(-1290$ to 32,000$)$ & 3993 (200 to 9500$)$ \\
\hline $\begin{array}{l}\text { Average Percent Change in ANC } \\
\text { (range) }\end{array}$ & $\begin{array}{l}1011 \%(33 \% \text { to } \\
53,500 \%)\end{array}$ & $\begin{array}{l}1365 \%(19 \% \text { to } \\
62,000 \%)\end{array}$ & $\begin{array}{l}728 \%(117 \% \text { to } \\
24,500 \%)\end{array}$ \\
\hline
\end{tabular}

\section{TABLE 4: Outcomes in different weight groups.}

ANC: Absolute neutrophil count; WBC: White blood cells.

\section{Discussion}

There have been limited reports examining whether $300 \mathrm{ug}$ of filgrastim is as efficacious as the recommended dose of $5 \mathrm{ug} / \mathrm{kg}$, particularly for patients with weights above $60 \mathrm{~kg}$. As such, this retrospective single-institution study aimed at examining whether patients receiving $300 \mathrm{ug}$ of filgrastim experience similar incidences of FN, neutropenia-related infections, and dose reductions or treatment delays as previous reports in the literature that prescribed patients a 5 $\mathrm{ug} / \mathrm{kg}$ dose. Based on our findings, patients in the medium weight cohort (that received a subtherapeutic dose) had similar WBC/ANC responses as well as neutropenia-related outcomes as compared to those in the low-weight cohort (that received the recommended dose). However, given the significantly higher infection rate among the high-weight group, patients with weights $>85 \mathrm{~kg}$ seem to have inferior outcomes with the administration of low-dose filgrastim.

There have been many other studies in the literature examining the impact of filgrastim and similar short-acting G-CSF analogs in reducing incidences of FN as well as hospitalizations relating to FN following administration at the recommended dose of $5 \mathrm{ug} / \mathrm{kg}$. A multicenter retrospective review with a cohort of patients with a wide range of cancer sites (i.e., lung, GI, GU, head and neck, etc.) treated with filgrastim for a median range of five days has previously reported $10.9 \%$ of patients experiencing FN-related hospitalizations (23/211) [13]. In one of the largest multicenter studies representing a variety of cancer sites conducted to date, patients receiving a mean of 5.2 and 3.7 days of consecutive filgrastim treatments in 2001 and 2003, respectively, were reported as having FN incidences of 5.3\% (31/583) and 7.3\% (63/868) [14]. Other retrospective studies as well as randomized trials examining the use of filgrastim and 
other short-acting G-CSFs for breast cancer patients receiving adjuvant or neoadjuvant chemotherapy reported incidences of FN in their cohorts ranging from 9.1\% to 18\% [15-17]. One study with similar findings to our own found FN incidence among Stage II to IV breast cancer patients treated with filgrastim (the majority receiving at least nine injections) to be $2.38 \%$ [18]. As such, the shorter course of low dose filgrastim administered among the medium weight cohort at least achieved similar rates of FN-related hospitalization (3.3\%) reports that have been published for patients receiving the recommended dose.

With regards to dose reductions and delay, approximately $6 \%, 5.5 \%$, and $8 \%$ of patients in the low, medium, and high weight groups required dose reductions and roughly $25 \%$ of patients among each of the weight groups had treatment delays. These findings also seem to be consistent with the literature. In a cohort study of 239 breast cancer patients that were administered filgrastim over a median of seven days (range: 4-10 days), 17\% of patients required a delay of treatment and 19\% required a dose reduction [19]. Another study examining the possible benefit of filgrastim among 1058 breast cancer patients reported that $18.5 \%$ and $42 \%$ of patients receiving filgrastim required a dose reduction or treatment delay, respectively [20]. In comparison to prior reports, $300 \mathrm{ug}$ of filgrastim for patients in our study with weights $<85 \mathrm{~kg}$ seemed to produce a non-inferior outcome.

As another area of interest, we analyzed the cost-savings of administering the two-day course of low dose of filgrastim as compared to a two-day course at the recommended dose that would necessitate the purchase of a 480 ug dose. Given that the medium weight group had similar clinical outcomes to both the low weight group in our study as well as other cohorts reported in the literature, we limited our analysis to this subset. Over 91 encounters for the medium weight cohort and assuming a wholesale cost of $\$ 1889.20$ for a 480 ug dose as compared to $\$ 1080$ for a 300 ug dose (representing a nearly $43 \%$ cost savings per dose), the total cost savings for this cohort alone translates to $\$ 147,274.40$. Given the small size of this cohort, these cost savings would no doubt have significant implications at a national level if non-inferiority is able to be further corroborated. Also, it is important to note that this analysis only takes into account potential savings from the lower dosage utilized but not from a two-day versus the typical multiple-day dosing.

There are limitations to our study that merit attention. First, the total patient cohort was small, especially when dividing among different weight groups, which limits both the power and generalizability of our findings. Second, there is the possibility of under-reporting, particularly with regards to incidences of FN and subsequent hospitalizations. Our institution serves as a tertiary care provider for a large geographic area, and as such patients seen in our outpatient clinics may have been admitted to other hospitals for FN that we were unable to account for in our analysis. Similarly, even if patients reported having been hospitalized for FN or infection following chemotherapy at outside institutions, we did not report on the severity and duration of FN-related hospitalizations. Also, the variety of cancer sites that the patients represented as well as varying chemotherapy regimens administered also limits the generalizability of our findings. Another limitation is the lack of control for other comorbid conditions that might have impacted the likelihood of infection or FN risk. It is also not ideal to have unequal time intervals between the repeat $\mathrm{CBC}$ and filgrastim administration, though roughly $68 \%$ of patients had a repeat CBC within roughly one standard deviation (roughly 3-10 days based on a mean of 6.6 days and a standard deviation of 3.2 days). Even though some patients did have repeat $\mathrm{CBC}$ outside of this time frame, we believe other reported outcomes such as infections and hospitalization are representative of immune response as well. Finally, the retrospective nature of our study and lack of inclusion and randomization between the lower dose of $300 \mathrm{ug}$ and the recommended $5 \mathrm{ug} / \mathrm{kg}$ among similar weight groups make comparison of outcomes between these two regimens difficult. 


\section{Conclusions}

The results of this retrospective single institution study have demonstrated non-inferior outcomes with regards to incidences of FN-related hospitalizations, infections, dose reductions, and treatment delays utilizing a two-day course of 300 ug of filgrastim for patients with weight $<85 \mathrm{~kg}$. Based on this observation, dosing filgrastim at 300 ug may be appropriate in this weight group with a roughly $43 \%$ cost reduction in our experience. Prospective randomized trials are warranted to validate these findings in a larger uniform cohort controlled for both disease site as well as chemotherapy received.

\section{Additional Information \\ Disclosures}

Human subjects: Consent was obtained by all participants in this study. Marshall University IRB issued approval 947237-2. Animal subjects: All authors have confirmed that this study did not involve animal subjects or tissue. Conflicts of interest: In compliance with the ICMJE uniform disclosure form, all authors declare the following: Payment/services info: All authors have declared that no financial support was received from any organization for the submitted work. Financial relationships: All authors have declared that they have no financial relationships at present or within the previous three years with any organizations that might have an interest in the submitted work. Other relationships: All authors have declared that there are no other relationships or activities that could appear to have influenced the submitted work.

\section{References}

1. Caggiano V, Weiss RV, Rickert TS, et al.: Incidence, cost, and mortality of neutropenia hospitalization associated with chemotherapy. Cancer. 2005, 103:1916-1924. 10.1002/cncr.20983

2. Kuderer NM, Dale DC, Crawford J, et al.: Mortality, morbidity, and cost associated with febrile neutropenia in adult cancer patients. Cancer. 2006, 106:2258-2266. 10.1002/cncr.21847

3. Falandry C, Campone M, Cartron G, et al.: Trends in G-CSF use in 990 patients after EORTC and ASCO guidelines. Eur J Cancer. 2010, 46:2389-2398. 10.1016/j.ejca.2010.04.031

4. Freifeld AG, Bow EJ, Sepkowitz KA, et al.: Clinical practice guideline for the use of antimicrobial agents in neutropenic patients with cancer: 2010 update by the Infectious Diseases Society of America. Clin Infect Dis. 2011, 52:56-93. 10.1093/cid/cir073

5. Smith TJ, Bohlke K, Lyman GH, et al.: Recommendations for the use of WBC growth factors: American Society of Clinical Oncology Clinical Practice Guideline Update. J Clin Oncol. 2015, 33:3199-3212. 10.1200/JCO.2015.62.3488

6. Aapro MS, Bohlius J, Cameron DA, et al.: 2010 update of EORTC guidelines for the use of granulocyte-colony stimulating factor to reduce the incidence of chemotherapy-induced febrile neutropenia in adult patients with lymphoproliferative disorders and solid tumours. Eur J Cancer. 2011, 47:8-32. 10.1016/j.ejca.2010.10.013

7. Crawford J, Caserta C, Roila F, et al.: Hematopoietic growth factors: ESMO clinical practice guidelines for the applications. Ann Oncol. 2010, 21:248-251. 10.1093/annonc/mdq195

8. Crawford J, Armitage J, Balducci L, et al.: Myeloid growth factors. J Natl Compr Canc Netw. 2013, 11:1266-1290. 10.6004/jnccn.2013.0148

9. Hartmann LC, Tschetter LK, Habermann TM, et al.: Granulocyte colony-stimulating factor in severe chemotherapy-induced afebrile neutropenia. N Engl J Med. 1997, 336:1776-1780. 10.1056/NEJM199706193362502

10. Gourzoulidis G, Kourlaba G, Apostolidis J, et al.: Cost-effectiveness analysis of granulocyte colony-stimulating factors for the prophylaxis of chemotherapy-induced febrile neutropenia in patients with non-Hodgkin lymphoma in Greece. Value Health. 2015, 18:457. 10.1016/j.jval.2015.09.1173

11. Leonard RC, Mansi JL, Keerie C, et al.: A randomised trial of secondary prophylaxis using granulocyte colony-stimulating factor ('SPROG' trial) for maintaining dose intensity of 
standard adjuvant chemotherapy for breast cancer by the Anglo-Celtic Cooperative Group and NCRN. Ann Oncol. 2015, 26:2437-2441. 10.1093/annonc/mdv389

12. Trautman H, Szabo E, Lo-Coco F, et al.: Budget impact analysis of treating chemotherapy patients with health care provider-administered Tbo-Filgrastim, Filgrastim-Sndz, and Filgrastim in the United States. Blood. 2016, 128:2407.

13. Almenar Cubells D, Bosch Roig C, Jiménez Orozco E, et al.: Effectiveness of daily versus nondaily granulocyte colony-stimulating factors in patients with solid tumours undergoing chemotherapy: a multivariate analysis of data from current practice. Eur J Cancer Care (Engl). 2013, 22:400-412. 10.1111/ecc.12043

14. Morrison VA, Wong M, Hershman D, et al.: Observational study of the prevalence of febrile neutropenia in patients who received filgrastim or pegfilgrastim associated with 3-4 week chemotherapy regimens in community oncology practices. J Manag Care Pharm. 2007, 13:337-348. 10.18553/jmcp.2007.13.4.337

15. Britton JA, Khan AE, Rohrmann S, et al.: Anthropometric characteristics and non-Hodgkin's lymphoma and multiple myeloma risk in the European prospective investigation into cancer and nutrition (EPIC). Haematologica. 2008, 93:1666-1677. 10.3324/haematol.13078

16. Schippinger W, Holub R, Dandachi N, et al.: Frequency of febrile neutropenia in breast cancer patients receiving epirubicin and docetaxel/paclitaxel with colony-stimulating growth factors: a comparison of filgrastim or lenograstim with pegfilgrastim. Oncology. 2006, 70:290-293. $10.1159 / 000094890$

17. von Minckwitz G, Kümmel S, du Bois A, et al.: Pegfilgrastim +/- ciprofloxacin for primary prophylaxis with TAC (docetaxel/doxorubicin/cyclophosphamide) chemotherapy for breast cancer. Results from the GEPARTRIO study. Ann Oncol. 2008, 19:292-298. 10.1093/annonc/mdm438

18. Hegg R, Mattar A, Matos-Neto JN, et al.: A phase III, randomized, non-inferiority study comparing the efficacy and safety of biosimilar filgrastim versus originator filgrastim for chemotherapy-induced neutropenia in breast cancer patients. Clinics (Sao Paulo). 2016, 71:586-592. 10.6061/clinics/2016(10)06

19. Altwairgi AK, Hopman WM, Mates M: Real-world impact of granulocyte-colony stimulating factor on febrile neutropenia. Curr Oncol. 2013, 20:171-179. 10.3747/co.20.1306

20. Kourlaba G, Dimopoulos MA, Pectasides D, et al.: Comparison of filgrastim and pegfilgrastim to prevent neutropenia and maintain dose intensity of adjuvant chemotherapy in patients with breast cancer. Support Care Cancer. 2015, 23:2045-2051. 10.1007/s00520-014-2555-y 